\title{
ON QUASI-ANALYTIC VECTORS FOR DISSIPATIVE OPERATORS
}

\section{MINORU HASEGAWA}

ABSTRACT. In this note we shall prove that a closed dissipative operator $A$ with dense domain in a hilbert space $H$ generates a contraction semigroup if the set

$$
\left\{A^{k} x k=0,1,2, \cdots, x \text { is quasi-analytic }\right\}
$$

is total in $H$.

1. Introduction. Let $H$ be a hilbert space with inner product $(x, y)\left(\|x\|=(x, x)^{1 / 2}\right)$ and $A$ be a linear operator with domain $D(A)$ and range $R(A)$ in $H$. If a vector $x \in \cap D\left(A^{n}\right)$ satisfies

$$
\sum_{n=1}^{\infty}\left(\inf _{k \geq n} m_{k}\right)^{-1}=\infty \quad \text { where }\left\|A^{n} x\right\|^{1 / n}=O\left(m_{n}\right)
$$

then $x$ is called a quasi-analytic vector for $A$.

In [2] A. E. Nussbaum has introduced the notion of quasi-analytic vectors and has shown, using a theorem of M. Naimark, that a closed symmetric operator $A$ is selfadjoint if and only if the set $\left\{A^{k} x\right.$; $k=0,1,2, \cdots, x$ is quasi-analytic $\}$ is total in $H$.

Now we introduce dissipative operators which have been extensively studied by R. S. Phillips (see, e.g., [3]).

A linear operator $A$ acting in $H$ is said to be dissipative if

$$
(A x, x)+(x, A x) \leqq 0 \quad(x \in D(A)) .
$$

In this note we shall show that the above Nussbaum condition is also sufficient for a closed dissipative operator with dense domain to generate a strongly continuous semigroup of contraction operators. For simplicity this semigroup is called a contraction semigroup.

2. Dissipative operators. In the sequel we need the following fundamental result (see [3]).

TheOREM. Let $A$ be a dissipative operator with dense domain in $H$. Then $A$ generates a contraction semigroup if and only if $R(k I-A)=H$ for some $k>0$.

Received by the editors August 11, 1970.

AMS 1969 subject classifications. Primary 4750.

Key words and phrases. Quasi-analytic vectors, dissipative operators, contraction semigroups. 
Proof. Since $A$ is dissipative, we see that, for any $x \in D(A)$,

$$
b\|x\|^{2} \leqq \operatorname{Re}(b(x, x)-(A x, x)) \leqq\|(b I-A) x\|\|x\|
$$

and that $(b I-A)^{-1}$ exists. By hypothesis, $R(k I-A)=H$ so that $k$ is in the resolvent set of $A$ and $\left\|(k I-A)^{-1}\right\| \leqq k^{-1}$. Moreover, using the well-known method based on the formula

$$
(b I-A)^{-1}=(k I-A)^{-1}\left(I+(b-k)(k I-A)^{-1}\right)^{-1},
$$

we have that $R(b I-A)=H$ and $\left\|(b I-A)^{-1}\right\| \leqq b^{-1}$ for any $b>0$. Thus the assertion follows from the Hille-Yosida theorem.

THEOREM 1. Let $k>0$ and $A$ be a closed dissipative operator. We define the operator $A_{k}$ on the linear manifold $D_{k}$, generated by $D(A)$ and the orthogonal complement $R(k I-A) \perp$ of $R(k I-A)$, by

$$
A_{k} x=A y-k z \text { for } x=y+z,
$$

where $y \in D(A)$ and $z \in R(k I-A) \perp$. Then $A_{k}$ is the generator of $a$ contraction semigroup.

Proof. Since $A$ is dissipative, we have that $D(A) \cap R(k I-A) \perp$ $=\{0\}$. It is easy to see that $A_{k}$ is linear and dissipative:

$$
\left(A_{k} x, x\right)+\left(x, A_{k} x\right)=(A y, y)+(y, A y)-2 k\|z\|^{2} .
$$

Now we note that, since $A$ is closed dissipative, the closure of $R(k I-A)$ is equal to $R(k I-A)$. It follows from $\left(k I-A_{k}\right) x$ $=(k I-A) y+2 k z$ that $R\left(k I-A_{k}\right)=H$. Thus the assertion is proved.

Theorem 2. A dissipative operator has an extension which is the generator of a contraction semigroup if and only if it has a closed extension.

Proof. Since it is known that the generator of a contraction semigroup is closed, the condition is necessary.

Now we note that the closure of a dissipative operator is also dissipative. Thus it follows from Theorem 1 that the condition is sufficient.

REMARK 1. It is known that any dissipative operator with dense domain has an extension which is the generator of a contraction semigroup (see [3]). We prove here this fact as a corollary of Theorem 2. In fact, letting $U(k)$ be the unique extension of $(k I-A)^{-1}$ which satisfies $\|U(k)\| \leqq k^{-1}$ on the closure of $R(k I-A)$, if $x_{n} \rightarrow 0$ and $A x_{n} \rightarrow y$ as $n \rightarrow \infty$, then we have that

$$
\left\|x_{n}+U(k) y\right\| \leqq k^{-1}\left\|(k I-A) x_{n}+y\right\|
$$


and that $U(k) y=0$ for $k=1,2, \cdots$. Since $D(A)$ is dense in $H$, it is easy to see that $k U(k) y \rightarrow y$ as $k \rightarrow \infty$. It follows that $y=0$ and $A$ has a closed extension. Thus the assertion follows from Theorem 2 .

\section{Main result.}

THEOREM 3. Let $A$ be a closed dissipative operator with dense domain. If the set $\left\{A^{k} x ; k=0,1,2, \cdots, x\right.$ is quasi-analytic $\}$ is total in $H$, then $A$ is the generator of a contraction semigroup.

Proof. It is sufficient to prove that $R(k I-A)=H$ for some $k>0$. Here we use the argument of Theorem 1 . Assume the contrary and suppose that the closure of $R(I-A)$ and $R(2 I-A)$ are properly contained in $H$. Since $D(A)$ is dense in $H$, we see that $R(I-A) \perp$ $\cap R(2 I-A) \perp=\{0\}$ and that $D_{1}$ and $D_{2}$ are different sets containing $D(A)$ properly. Let $\{T(t) ; t \geqq 0\}$ and $\{S(t) ; t \geqq 0\}$ be the contraction semigroups generated by $A_{1}$ and $A_{2}$ respectively. Define, for any quasi-analytic vector $x$ for $A$ and for any $y \in H$,

$$
\begin{aligned}
f(t) & =(T(t) x-S(t) x, y), & & t \geqq 0, \\
& =0, & & t<0 .
\end{aligned}
$$

Then

$$
\begin{aligned}
\left|f^{(n)}(t)\right|^{1 / n} & =\left|\left(T(t) A^{n} x-S(t) A^{n} x, y\right)\right|^{1 / n} \\
& \leqq\left(2\|y\|\left\|A^{n} x\right\|\right)^{1 / n}=O\left(m_{n}\right)
\end{aligned}
$$

and

$$
\sum_{n=1}^{\infty}\left(\inf _{k \geqq n} m_{k}\right)^{-1}=\infty
$$

By the Carleman theorem, $f(t)$ is quasi-analytic and $f(t) \equiv 0$. Thus we see that, for any quasi-analytic vector $x$ for $A$,

$$
T(t) A^{k} x=S(t) A^{k} x \quad(k=0,1,2, \cdots) .
$$

It follows from the hypothesis that $T(t) \equiv S(t)$ and that $A_{1} \equiv A_{2}$ which is a contradiction. Thus the assertion is proved.

REMARK 2. Here we shall prove the Nussbaum theorem mentioned in the introductory part as an application of Theorem 3.

It is easy to see that $A$ is symmetric if and only if $i A$ is conservative, that is,

$$
(i A x, x)+(x, i A x)=0 \quad(x \in D(A)) .
$$

By Theorem $3, i A$ and $-i A$ generate contraction semigroups. Thus 
$i A$ is the generator of a group of unitary operators and hence $A$ is selfadjoint.

REMARK 3. Let $H=L^{2}(0, \infty)$ and define $\left\{T_{t} ; t \geqq 0\right\}$ by

$$
\begin{aligned}
T_{t} u(x) & =u(x-t), & x \geqq t, \\
& =0, & 0 \leqq x<t .
\end{aligned}
$$

This example was used by E. Nelson [1] to show that the only analytic vector for the generator $A$ of this contraction semigroup is 0 . We now remark that the only quasi-analytic vector for $A$ is also 0 . Thus the converse of Theorem 3 does not hold in general.

REMARK on TheOREM 1 . This extension $A_{k}$ of a closed dissipative operator $A$, in case of $A$ being densely defined, was obtained by S. G. Krel̆n in Linear differential equations in a Banach space, Izdat. "Nauka", Moscow, 1967. (Russian) The author wishes to express his hearty thanks to Professor I. Miyadera and Mr. N. Okazawa for calling his attention to this.

\section{REFERENCES}

1. E. Nelson, Analytic vectors, Ann. of Math. (2) 70 (1959), 572-615. MR 21 \#5901.

2. A. E. Nussbaum, Quasi-analytic vectors, Ark. Mat. 6 (1965), 179-191. MR 33 \#3105.

3. R. S. Phillips, Dissipative operators and hyperbolic systems of partial differential equations, Trans. Amer. Math. Soc. 90 (1959), 193-254. MR 21 \#3669.

Tokyo Metropolitan University, Tokyo 158, Japan 